\title{
Surgery for Normal Pressure Glaucoma-Is There a Role for Minimally Invasive Glaucoma Surgery Before Filtering Surgery?
}

\author{
An Expert Interview with Leonard K Seibold
}

Sue Anschutz-Rodgers Eye Center, Aurora, CO, USA

DOI: https://doi.org/10.17925/USOR.2019.12.2.57

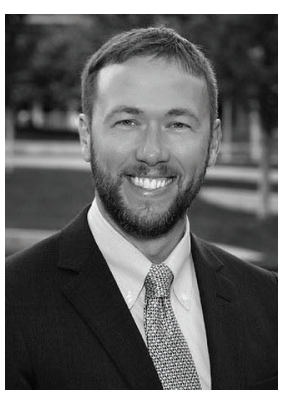

\section{Leonard $K$ Seibold}

Dr Seibold is an associate professor in ophthalmology at the University of Colorado school of Medicine and is the director of the glaucoma fellowship. After completion of medical school at the University of Oklahoma, Dr Seibold completed his ophthalmology residency training and glaucoma fellowship at the University of Colorado. He specializes in the medical and surgical treatment of adult and pediatric glaucomas as well as the surgical treatment for cataracts. His research interests include 24-Hour IOP monitoring and novel minimally invasive glaucoma surgery procedures.

\section{Keywords}

Normal pressure glaucoma, minimally invasive glaucoma surgery, MIGS

Disclosure: Leonard $\mathrm{K}$ Seibold is a consultant to New World Medical and Allergan, and has received research support from Glaukos and Allergan.

Review Process: This is an opinion piece and has therefore not undergone the journal's usual peer review process.

Authorship: All named authors meet the International Committee of Medical Journal Editors (ICMJE) criteria for authorship of this manuscript, take responsibility for the integrity of the work as a whole, and have given final approval to the version to be published.

Received: November 27, 2019

Published Online: December 12, 2019

Citation: US Ophthalmic Review, 2019;12(2):57-8

Corresponding Author: Leonard K Seibold, Associate Professor, Glaucoma and Cataracts and Director of Glaucoma Fellowship, Sue AnschutzRodgers Eye Center, 1675 Aurora Court, Aurora, CO 800451, USA. E: leonard.seibold@cuanschutz.edu

Support: No funding was received in

the publication of this article.
$\mathrm{N}$ ormal tension glaucoma (NTG), also known as normal pressure or low tension glaucoma, is characterized by damage to the optic nerve without raised intraocular pressure (IOP)., ${ }^{1,2}$ For many years ophthalmologists have found the diagnosis of NTG challenging, but following the Collaborative Normal Tension Glaucoma Study, it is generally accepted that IOP reduction can prevent or slow visual field (VF) loss in NTG although patient response is varied. ${ }^{3}$ In recent years, minimally-invasive glaucoma surgeries (MIGS) has emerged as an attractive treatment option for patients with open angle glaucoma. ${ }^{4}$ However, it is not known whether MIGS may be beneficial in the treatment of patients with NTG. In an expert interview, Professor Seibold discusses the potential role of MIGS in patients with NTG.

\section{Q. Could you tell us a little about normal pressure glaucoma and its prevalence?}

Normal tension glaucoma (NTG) is generally characterized by the same optic nerve cupping, retinal nerve fiber layer thinning, and visual field loss seen in primary open angle glaucoma (POAG), but with intraocular pressures (IOP) that remain less than $22 \mathrm{mmHg}$. The disease also has several unique features that further distinguish it from POAG, such as more frequent optic nerve hemorrhage, rim notching, and central visual field defects that occur earlier in the disease course. Disease prevalence can vary widely but is estimated to be between $0.5-3 \%$ worldwide.

\section{Q. What are the potential complications of filtering surgery in normal pressure glaucoma?}

Filtering surgery can result in a number of devastating complications no matter what type of glaucoma is being treated. These include endophthalmitis, choroidal hemorrhage, and hypotony related problems. Due to the very low target IOP levels in patients with NTG (often $<10 \mathrm{mmHg}$ ), surgeons must aggressively titrate aqueous outflow during trabeculectomy to achieve these levels. This increases the risk of hypotony related complications such as choroidal effusions, shallow or flat anterior chambers, and hypotony maculopathy in these patients. 


\section{Q. What is the potential role of MIGS in the treatment of normal pressure glaucoma?}

Minimally-invasive glaucoma surgery (MIGS) offers a number of alternative procedures aimed not only at lowering intraocular pressure but also at reducing glaucoma medication requirement. These procedures offer a surgical alternative to trabeculectomy that has a more favorable safety profile and faster recovery. Similarly for NTG patients, MIGS procedures have a potential role to better control IOP and/or reduce the medication burden either at the time of cataract surgery or as a standalone procedure in some cases. They may also delay a more invasive procedure such as trabeculectomy or glaucoma drainage device and their associated complications.

\section{Q. What are the limitations of MIGS in this treatment setting?}

Using the published MIGS literature in POAG or mixed glaucoma populations, most procedures have been shown to lower IOP to the low to mid-teens range on fewer medications. While a reduction in the number of eye drops may be helpful for an NTG patient, they can frequently have a treatment goal below this IOP range. Thus for patients with a target IOP $<10 \mathrm{mmHg}$, MIGS procedures will have limited success in achieving this treatment goal and trabeculectomy is likely the more effective procedure in that scenario.

\section{Q. What research has been performed in this field and what future studies are planned?}

Several MIGS studies have demonstrated successful outcomes in patient groups with a mean preoperative IOP $<22 \mathrm{mmHg}$. However, most MIGS procedures have not been studied exclusively in an NTG population thus far. Based on the mean postoperative IOP levels in a largely POAG population, clinicians can infer a final IOP in the range of $12-16 \mathrm{mmHg}$ is likely to be achieved. Future studies of clinical outcomes in NTG populations are certainly needed to better understand the role of these novel procedures in these patients. $\square$

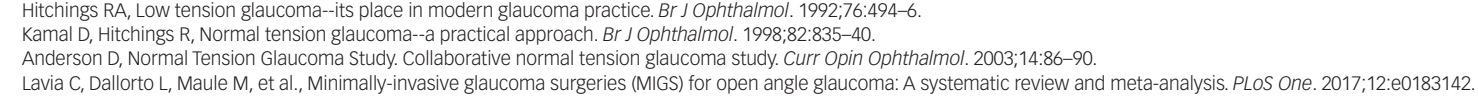

